\title{
Experimental Study of Totally Optimal Decision Rules
}

\author{
Mohammad Azad ${ }^{[0000-0001-9851-1420]}$ and Mikhail \\ Moshkov ${ }^{[0000-0003-0085-9483]}$ \\ King Abdullah University of Science and Technology (KAUST) \\ Computer, Electrical and Mathematical Sciences \& Engineering Division \\ Thuwal 23955-6900, Saudi Arabia \\ \{mohammad.azad, mikhail.moshkov\}@kaust.edu.sa
}

\begin{abstract}
In this paper, we experimentally study the existence of totally optimal decision rules which are optimal relative to the length and coverage simultaneously for nine decision tables from the UCI Machine Learning Repository. Totally optimal rules can be useful when we consider decision rules as a way for knowledge representation. We study not only exact but also approximate decision rules based on the three uncertainty measures: entropy, Gini index, and misclassification error. To investigate the existence of totally optimal rules, we use an extension of dynamic programming that allows us to make multi-stage optimization of decision rules relative to the length and coverage. Experimental results show that totally optimal decision rules exist in many cases. However, the behavior of graphs describing how the number of rows of decision tables with totally optimal decision rules depends on the accuracy of rules is irregular.
\end{abstract}

Keywords: Decision rule · Uncertainty measure · Length · Coverage · Totally optimal rule $\cdot$ Multi-stage optimization.

\section{Introduction}

Decision rules are widely used to solve the problems of prediction and knowledge representation $[8,9,13,14]$. When decision rules are considered as a way for knowledge representation, we would like to minimize their length (to make the rules more understandable) and to maximize their coverage (to make the rules more general).

In this paper, we experimentally investigate the existence of totally optimal decision rules that have the minimum length and the maximum coverage simultaneously.

We can use two tools for the study of totally optimal decision rules that are based on extensions of dynamic programming. These are (i) multi-stage optimization of decision rules relative to two criteria, and (ii) construction of the set of Pareto optimal points for bi-criteria optimization problem (totally optimal decision rule exists if and only if there is exactly one Pareto optimal 


\section{Azad and M. Moshkov}

point) [1]. In this paper, we use the first tool which is less time consuming. For experimenting, we use Dagger system created in KAUST to implement various extensions of dynamic programming $[2,3]$.

One of the main goals of this paper is to experimentally study how the existence of totally optimal decision rules depends on the accuracy of such rules. We work with nine decision tables from the UCI Machine Learning Repository [12]. We study not only exact but also approximate decision rules defined based on the three uncertainty measures: entropy ent, Gini index gini, and misclassification error me. We consider 101 values of the threshold $\alpha$ from 0.00 to 1.00 with the step 0.01 . This threshold describes the accuracy of decision rules: $\alpha=0.00$ means 100 percent accuracy (exact decision rules), and $\alpha=1.00$ means 0 percent accuracy.

Experimental results show that totally optimal decision rules exist in many cases. The behavior of graphs describing how the number of rows of decision tables with totally optimal decision rules depends on decision rule accuracy is irregular.

One of the main areas of applications of the obtained results (existence of totally optimal decision rules in many cases) is the rough set theory [10,15-17] in which decision rules are widely used. Another area of applications is the logical analysis of data (LAD) in which decision rules are known as patterns $[5-7,11]$.

Note that exact totally optimal decision rules were studied in $[1,4]$ both from theoretical and experimental points of view.

This paper consists of four sections. In Sect. 2, we consider main notions and tools. Section 3 is devoted to the consideration of experimental results. Section 4 contains short conclusions.

\section{Main Notions and Tools}

In this section, we discuss the notions of decision table, uncertainty measure, decision rule, cost function, and totally optimal decision rule. We consider also a way to prove the existence of totally optimal decision rules.

\subsection{Decision Tables and Uncertainty Measures}

A decision table is a rectangular table $T$ with $n \geq 1$ columns filled with numbers from the set $\omega=\{0,1,2, \ldots\}$ of nonnegative integers. Columns of the table are labeled with conditional attributes $f_{1}, \ldots, f_{n}$. Rows of the table are pairwise different, and each row is labeled with a number from $\omega$ which is interpreted as a decision (a value of the decision attribute $d$ ). Rows of the table are interpreted as tuples of values of conditional attributes. We denote by $\mathcal{T}$ the set of all decision tables.

A decision table is called empty if it has no rows. The table $T$ is called degenerate if it is empty or all rows of $T$ are labeled with the same decision. Let $D(T)$ be the set of decisions attached to rows of $T$. We denote by $N(T)$ the number of rows in the table $T$ and, for any $t \in \omega$, we denote by $N_{t}(T)$ the 
Experimental Study of Totally Optimal Decision Rules

number of rows of $T$ labeled with the decision $t$. By $\operatorname{mcd}(T)$ we denote the most common decision for $T$ which is the minimum decision $t_{0}$ from $D(T)$ such that $N_{t_{0}}(T)=\max \left\{N_{t}(T): t \in D(T)\right\}$. If $T$ is empty then $\operatorname{mcd}(T)=0$.

For any conditional attribute $f_{i} \in\left\{f_{1}, \ldots, f_{n}\right\}$, we denote by $E\left(T, f_{i}\right)$ the set of values of the attribute $f_{i}$ in the table $T$. We denote by $E(T)$ the set of conditional attributes for which $\left|E\left(T, f_{i}\right)\right| \geq 2$.

Let $T$ be a nonempty decision table. A subtable of $T$ is a table obtained from $T$ by removal of some rows. Let $f_{i_{1}}, \ldots, f_{i_{m}} \in\left\{f_{1}, \ldots, f_{n}\right\}$ and $a_{1}, \ldots, a_{m} \in \omega$. We denote by $T\left(f_{i_{1}}, a_{1}\right) \ldots\left(f_{i_{m}}, a_{m}\right)$ the subtable of the table $T$ containing the rows from $T$ which at the intersection with the columns $f_{i_{1}}, \ldots, f_{i_{m}}$ have numbers $a_{1}, \ldots, a_{m}$, respectively.

Let $\mathbb{R}$ be the set of real numbers. An uncertainty measure is a function $U: \mathcal{T} \rightarrow \mathbb{R}$ such that $U(T) \geq 0$ for any $T \in \mathcal{T}$, and $U(T)=0$ if and only if $T$ is a degenerate table. The following functions (we assume that, for any empty table, the value of each of the considered functions is equal to 0 ) are uncertainty measures:

- Entropy ent $(T)=-\sum_{t \in D(T)}\left(N_{t}(T) / N(T)\right) \log _{2}\left(N_{t}(T) / N(T)\right)$.

- Gini index $\operatorname{gini}(T)=1-\sum_{t \in D(T)}\left(N_{t}(T) / N(T)\right)^{2}$.

- Misclassification error $m e(T)=N(T)-N_{m c d(T)}(T)$.

\subsection{Decision Rules and Cost Functions}

Let $T$ be a decision table with $n$ conditional attributes $f_{1}, \ldots, f_{n}$ and $r=$ $\left(b_{1}, \ldots, b_{n}\right)$ be a row of $T$. A decision rule over $T$ is an expression of the kind

$$
\left(f_{i_{1}}=a_{1}\right) \wedge \ldots \wedge\left(f_{i_{m}}=a_{m}\right) \rightarrow t
$$

where $f_{i_{1}}, \ldots, f_{i_{m}} \in\left\{f_{1}, \ldots, f_{n}\right\}$, and $a_{1}, \ldots, a_{m}, t$ are numbers from $\omega$. It is possible that $m=0$. For the considered rule, we denote $T^{0}=T$, and if $m>0$ we denote $T^{j}=T\left(f_{i_{1}}, a_{1}\right) \ldots\left(f_{i_{j}}, a_{j}\right)$ for $j=1, \ldots, m$. We will say that the decision rule (1) covers the row $r$ if $r$ belongs to $T^{m}$, i.e., $b_{i_{1}}=a_{1}, \ldots, b_{i_{m}}=a_{m}$.

A decision rule (1) over $T$ is called a decision rule for $T$ if $t=\operatorname{mcd}\left(T^{m}\right)$, and either $m=0$, or $m>0$ and, for $j=1, \ldots, m, T^{j-1}$ is not degenerate, and $f_{i_{j}} \in E\left(T^{j-1}\right)$. A decision rule (1) for $T$ is called a decision rule for $T$ and $r$ if it covers $r$.

Let $U$ be an uncertainty measure and $\alpha$ be a real number such that $0 \leq \alpha \leq 1$. A decision rule (1) for $T$ is called a $(U, \alpha)$-decision rule for $T$ if $U\left(T^{m}\right) \leq \alpha U(T)$ and, if $m>0$, then $U\left(T^{j}\right)>\alpha U(T)$ for $j=0, \ldots, m-1$. A $(U, \alpha)$-decision rule (1) for $T$ is called a $(U, \alpha)$-decision rule for $T$ and $r$ if it covers $r$.

We now consider a notion of cost function for decision rules. This is a function $\psi(T, \rho)$ which is defined on pairs $T, \rho$, where $T$ is a nonempty decision table and $\rho$ is a decision rule for $T$, and has values from the set $\mathbb{R}$ of real numbers.

We will work with the following two cost functions for decision rules:

- The length $l(T, \rho)=l(\rho)$. The length of the rule (1) is equal to $m$.

- The coverage $c(T, \rho)$. The coverage of the rule (1) for table $T$ is equal to $N_{m c d\left(T^{m}\right)}\left(T^{m}\right)$. 


\subsection{Totally Optimal Decision Rules}

Let $T$ be a decision table, $r$ be a row of $T, U$ be an uncertainty measure, and $\alpha$ be a real number such that $0 \leq \alpha \leq 1$.

We denote by $l^{U, \alpha}(T, r)$ the minimum length of a $(U, \alpha)$-decision rule for $T$ and $r$. By $c^{U, \alpha}(T, r)$ we denote the maximum coverage of a $(U, \alpha)$-decision rule for $T$ and $r$. A $(U, \alpha)$-decision rule $\rho$ for $T$ and $r$ is called a totally optimal $(U, \alpha)$ decision rule for $T$ and $r$ relative to the length and coverage if $l(\rho)=l^{U, \alpha}(T, r)$ and $c(T, \rho)=c^{U, \alpha}(T, r)$, i.e., $\rho$ is optimal relative to $c$ and $l$ simultaneously.

We now describe how to recognize the existence of a $(U, \alpha)$-decision rule for $T$ and $r$ which is a totally optimal $(U, \alpha)$-decision rule for $T$ and $r$ relative to the length and coverage.

First, we apply to $T$ and $r$ the procedure of optimization relative to $c$ [1]. As a result, we obtain the number $c^{U, \alpha}(T, r)$. Next, we sequentially apply to $T$ the procedures of optimization relative to the length $l$ and coverage $c$ [1]. As a result, we obtain the number $c_{l}^{U, \alpha}(T, r)$ which is the maximum coverage among all $(U, \alpha)$-decision rules for $T$ and $r$ which have the minimum length (see [1]). One can show that a totally optimal $(U, \alpha)$-decision rule for $T$ and $r$ relative to the length and coverage exists if and only if $c_{l}^{U, \alpha}(T, r)=c^{U, \alpha}(T, r)$.

\section{Experimental Results}

In this section, we consider results of computer experiments with nine decision tables from [12]. Before the experimental work, some preprocessing procedures are performed. A conditional attribute is removed if it has unique value for each row. The missing value for an attribute is filled up with the most common value for this attribute. In some tables, there are equal rows with, possibly, different decisions. In this case, each group of equal rows is replaced with a single row from the group with the most common decision for this group. As a result, we obtain consistent decision tables without missing values (a decision table is called consistent if it has no equal rows with different decisions).

Table 1. Decision tables used in experiments.

\begin{tabular}{|l|r|r|}
\hline Decision table & Rows & Attributes \\
\hline BALANCE-SCALE & 625 & 5 \\
BREAST-CANCER & 266 & 10 \\
CARS & 1728 & 7 \\
HAYES-ROTH-DATA & 69 & 5 \\
HOUSE-VOTES-84 & 279 & 17 \\
LYMPHOGRAPHY & 148 & 19 \\
SOYBEAN-SMALL & 47 & 36 \\
TIC-TAC-TOE & 958 & 10 \\
ZOO-DATA & 59 & 17 \\
\hline
\end{tabular}


The nine decision tables from [12] used in experiments are described in Table 1 . The first column 'Decision table' refers to the name of the decision table from [12], the second column 'Rows' refers to the number of rows, and the last column 'Attributes' refers to the number of conditional attributes.

For each decision table $T$, each row $r$ of $T$, each uncertainty measure $U \in$ $\{$ ent, gini, me $\}$, and each $\alpha \in\{0.00,0.01,0.02, \ldots, 1.00\}$, we check if there exists a totally optimal $(U, \alpha)$-decision rule for $T$ and $r$ relative to the length and coverage. We denote $\operatorname{Tot}_{U}(T, \alpha)$ the number of rows $r$ in the decision table $T$ for each of which there exists a totally optimal $(U, \alpha)$-decision rule for $T$ and $r$ relative to the length and coverage. By $\operatorname{Tot}_{U}(\alpha)$ we denote the sum of numbers $\operatorname{Tot}_{U}(T, \alpha)$ for all nine considered decision tables $T$.

The results of experiments can be found in Figs. 1, 2, and 3 for uncertainty measures ent, gini, and me, respectively. In each figure, the graph of the function $\operatorname{Tot}_{U}(\alpha)$ is depicted for the uncertainty measure $U$ considered in this figure.

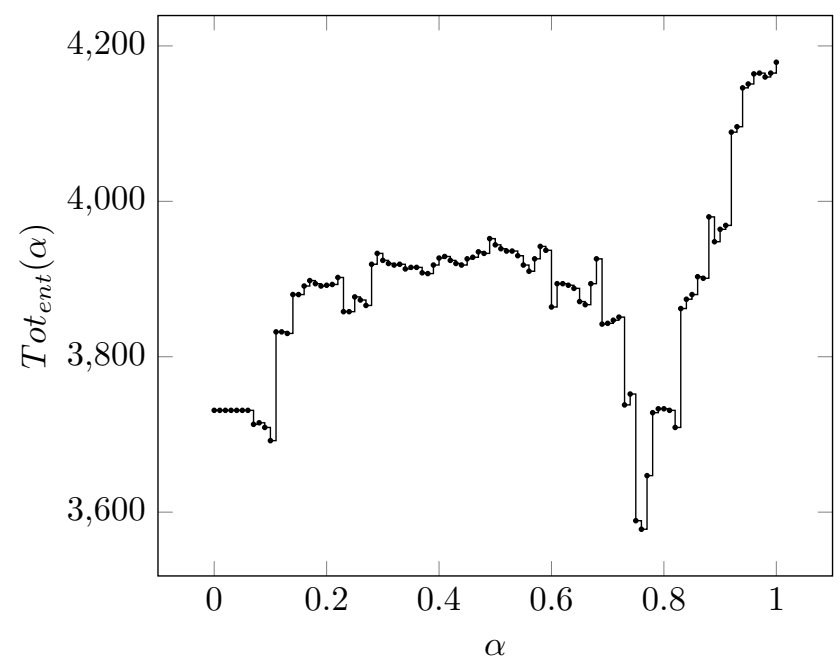

Fig. 1. Number of rows of decision tables with totally optimal decision rules for uncertainty measure ent.

The total number of rows in the considered decision tables is equal to 4179 . The minimum values of the functions $\operatorname{Tot}_{\text {ent }}(\alpha), \operatorname{Tot}_{\text {gini }}(\alpha)$, and $\operatorname{Tot}_{m e}(\alpha)$ are equal to 3578,3571 , and 3060 , respectively. It means that, for each of the considered value of $\alpha$, more than $85 \%$ of rows have totally optimal decision rules for the uncertainty measures ent and gini, and more than $73 \%$ of rows have totally optimal decision rules for the uncertainty measure me. The obtained results show that totally optimal decision rules exist in many cases. However, the behavior of graphs in Figs. 1-3 describing how the number of rows with totally optimal decision rules depends on the accuracy of rules is irregular. 
M. Azad and M. Moshkov

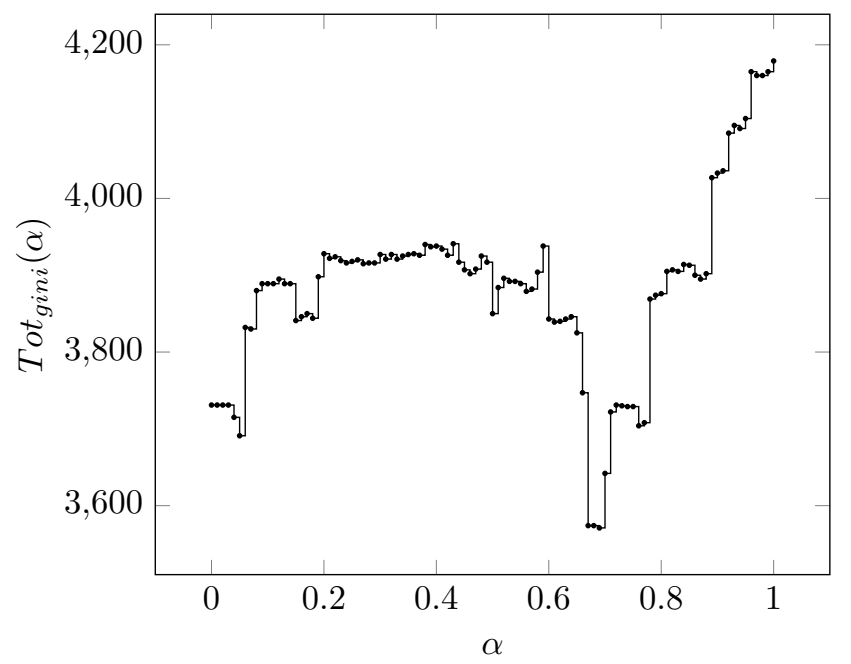

Fig. 2. Number of rows of decision tables with totally optimal decision rules for uncertainty measure gini.

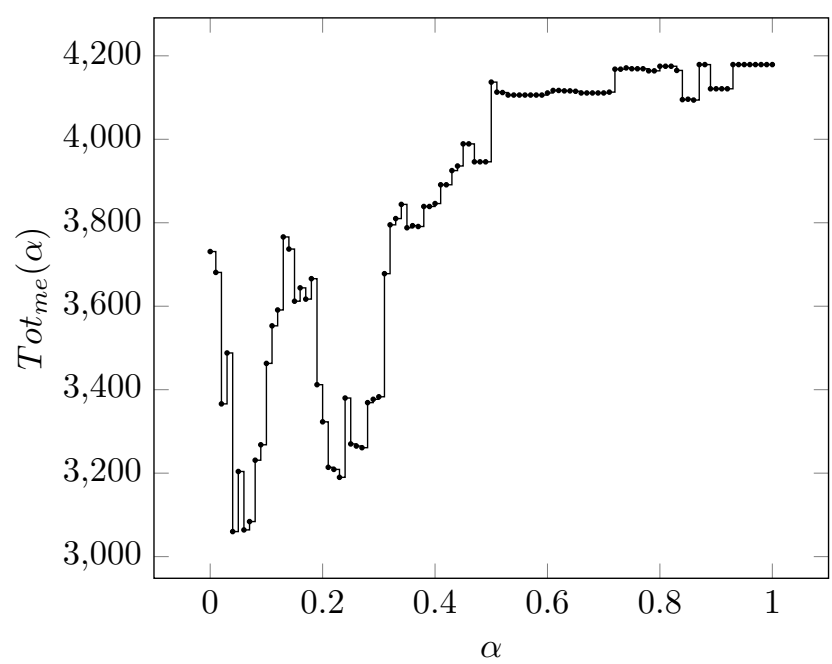

Fig. 3. Number of rows of decision tables with totally optimal decision rules for uncertainty measure me. 
Experimental Study of Totally Optimal Decision Rules

\section{Conclusions}

In this paper, we experimentally studied the existence of totally optimal decision rules (exact and approximate) for nine decision tables from the UCI Machine Learning Repository [12]. We showed that the totally optimal decision rules exist in many cases. In the future, we are planning to continue the study of totally optimal rules since they can be useful in applications related to knowledge representation. In particular, we are planning to use in the experiments not only whole datasets but also their samples to understand the stability of the obtained results.

\section{Acknowledgments}

Research reported in this publication was supported by King Abdullah University of Science and Technology (KAUST).

The authors are greatly indebted to the anonymous reviewer for useful comments.

\section{References}

1. AbouEisha, H., Amin, T., Chikalov, I., Hussain, S., Moshkov, M.: Extensions of Dynamic Programming for Combinatorial Optimization and Data Mining, Intelligent Systems Reference Library, vol. 146. Springer (2019)

2. Alkhalid, A., Amin, T., Chikalov, I., Hussain, S., Moshkov, M., Zielosko, B.: Dagger: a tool for analysis and optimization of decision trees and rules. In: Ficarra, F.V.C., Kratky, A., Veltman, K.H., Ficarra, M.C., Nicol, E., Brie, M. (eds.) Computational Informatics, Social Factors and New Information Technologies: Hypermedia Perspectives and Avant-Garde Experiencies in the Era of Communicability Expansion, pp. 29-39. Blue Herons (2011)

3. Alkhalid, A., Amin, T., Chikalov, I., Hussain, S., Moshkov, M., Zielosko, B.: Optimization and analysis of decision trees and rules: dynamic programming approach. International Journal of General Systems 42(6), 614-634 (2013)

4. Amin, T., Moshkov, M.: Totally optimal decision rules. Discrete Applied Mathematics 236, 453-458 (2018)

5. Bonates, T.O., Hammer, P.L., Kogan, A.: Maximum patterns in datasets. Discrete Applied Mathematics 156(6), 846-861 (2008)

6. Boros, E., Hammer, P.L., Ibaraki, T., Kogan, A.: Logical analysis of numerical data. Math. Programming 79, 163-190 (1997)

7. Boros, E., Hammer, P.L., Ibaraki, T., Kogan, A., Mayoraz, E., Muchnik, I.: An implementation of logical analysis of data. IEEE Transactions of Knowledge and Data Engineering 12, 292-306 (2000)

8. Fürnkranz, J.: Separate-and-conquer rule learning. Artif. Intell. Rev. 13(1), 3-54 (1999)

9. Fürnkranz, J., Gamberger, D., Lavrac, N.: Foundations of Rule Learning. Cognitive Technologies, Springer, Heidelberg (2012)

10. Góra, G., Wojna, A.: RIONA: A new classification system combining rule induction and instance-based learning. Fundam. Inform. 51(4), 369-390 (2002) 


\section{Azad and M. Moshkov}

11. Hammer, P.L., Kogan, A., Simeone, B., Szedmák, S.: Pareto-optimal patterns in logical analysis of data. Discrete Applied Mathematics 144(1-2), 79-102 (2004)

12. Lichman, M.: UCI Machine Learning Repository. University of California, Irvine, School of Information and Computer Sciences (2013), http://archive.ics.uci.edu/ml

13. Michalski, R.S., Pietrzykowski, J.: iAQ: a program that discovers rules. In: 22nd AAAI Conference on Artificial Intelligence, AI Video Competition (2007)

14. Mingers, J.: Expert systems - rule induction with statistical data. Journal of the Operational Research Society 38, 39-47 (1987)

15. Pawlak, Z.: Rough Sets - Theoretical Aspect of Reasoning About Data. Kluwer Academic Publishers, Dordrecht (1991)

16. Pawlak, Z., Skowron, A.: Rudiments of rough sets. Information Sciences 177(1), 3-27 (2007)

17. Sikora, M.: Decision rule-based data models using TRS and NetTRS - methods and algorithms. In: Peters, J.F., Skowron, A. (eds.) Trans. Rough Sets XI, Lecture Notes in Computer Science, vol. 5946, pp. 130-160. Springer (2010) 